\title{
Change Sensor Topology When Needed: How to Efficiently Use System Resources in Control and Estimation Over Wireless Networks
}

\author{
Ling Shi*, Karl Henrik Johansson ${ }^{\dagger}$ and Richard M. Murray*
}

\begin{abstract}
New control paradigms are needed for large networks of wireless sensors and actuators in order to efficiently utilize system resources. In this paper we consider when feedback control loops are formed locally to detect, monitor, and counteract disturbances that hit a plant at random instances in time and space. A sensor node that detects a disturbance dynamically forms a local multi-hop tree of sensors and fuse the data into a state estimate. It is shown that the optimal estimator over a sensor tree is given by a Kalman filter of certain structure. The tree is optimized such that the overall transmission energy is minimized but guarantees a specified level of estimation accuracy. A sensor network reconfiguration algorithm is presented that leads to a suboptimal solution and has low computational complexity. A linear control law based on the state estimate is applied and it is argued that it leads to a closed-loop control system that minimizes a quadratic cost function. The sensor network reconfiguration and the feedback control law are illustrated on an example.
\end{abstract}

\section{INTRODUCTION}

Control over large resource-constrained infrastructures requires new design paradigms beyond traditional sampleddata control. Difficulties that are inherit of these systems are (i) lack of global synchronization, (ii) constrained communication capabilities, and (iii) limited energy resources. (i) In most cases a centralized control strategy is unrealistic for these systems, since network nodes are executed asynchronously. Instead local control objectives should be defined that lead to that the desired global task is accomplished. (ii) Communication between network nodes is limited, particularly, if nodes are located physically far way from each other. It takes time to transfer information from one node to another, and in many cases this time increases if the information needs to be reliably delivered. (iii) An increasing number of nodes are battery powered. To extend the life time of such nodes, it is important to limit the amount of communication and computation they do.

The main contribution of this paper is to tackle these networked control problems by letting the communication topology adapt to the control task. We propose a new control structure such that if a local disturbance hits the system, sensors close to the event inform a local controller to make a decision of the action to take. To extend the battery lifetime

*: Control and Dynamical Systems, California Institute of Technology, Pasadena, CA 91106. Email: \{shiling, murray\}@cds.caltech.edu.

$\dagger$ : School of Electrical Engineering, Royal Institute of Technology, Stockholm, Sweden. Email: kallej@ee.kth.se.

Tel: (626) 395-2313, Fax: (626) 395-6170.

The work by L. Shi and R. M. Murray is supported in part by AFOSR grant FA9550-04-1-0169. The work by K. H. Johansson was supported by the Swedish Research Council and the Swedish Foundation for Strategic Research. of the wireless sensor network, data are communicated over a multi-hop wireless network, instead of a single-hop network. The feedback control architecture is illustrated in Figure 1. The quality of the state estimate used in the control node depends not only on the sensor quality but also on the communication delay, i.e., the number of hops the sensor reading needs to take until it reaches the fusion center (the black dot in Figure 1). Many short hops takes longer time than the few long hops. On the other hand, fewer hops require larger transmission power since the required transmission grows rapidly with the distance between the wireless nodes. Hence, there is a trade-off between state estimation quality and energy efficiency. The solution we propose is to optimize the network path for the sensor data such that the overall transmission energy is minimized, but guarantees a certain level of estimation accuracy. The resulting local sensor topology has the structure of a tree for which the fusion center is the root. We also propose a tree reconfiguration algorithm so that if sensor node failure happens, or new sensor joins, or existing sensor leaves to serve other applications, the tree can be reformed dynamically which increases robustness of the overall system. When the plant is given by a linear system, the optimal estimator is given by a Kalman filter with extra memory due to the communication delays. A control law is derived based on linear quadratic control theory taking into account the achieved estimation error covariance. The proposed estimation and control scheme scales well with network size, because of the local nature of the implementation and execution.

There are several potential application areas of the work presented in this paper, including building automation, environmental monitoring, industrial automation, power distribution, and transportation systems.

Some work related to this paper is described next. One way to deal with the problem of asynchronous generation of sensor data is to use event-triggered control instead of conventional time-triggered control [1], [2]. How to efficiently encode control information for event-triggered control over communication channels with severe bandwidth limitations is discussed in [3].

Kalman filtering under certain information constraints, such as decentralized implementation, has been extensively studied [4]. Implementations for which the computations are distributed among network nodes is considered in [5]-[7] Kalman filtering over lossy networks is considered in [8], [9]. The interaction between Kalman filtering and how data is routed on a network seems to be less studied. Routing of data packets in networks are typically done based on the distance 
to the receiver node [10]. Some recent work addresses how to couple data routing with the sensing task using information theoretic measures [11].

For control over wireless sensor networks, the experienced delays and packet losses are important parameters. Randomized routing protocols that gives probabilistic guarantees on delay and loss are proposed in [12], [13]. A compensation scheme in the controller for the variations on the transport layer that such routing protocols give rise is presented in [14]. A robust control approach to control over multihop networks is discussed in [15]. A general cross-layer approach to control and data routing seems to be an open and rather difficult topic due to many practical constraints. Our approach is different in that we make the assumption that a tree-structured sensor topology with certain properties can be superimposed on the sensor network. The routing of individual packets is not considered, but instead a number of paths are dynamically established between the sensor nodes and the controller.

The rest of the paper is organized as follows. The problem setup is described in Section II. Some definitions and preliminary facts on Kalman filter is provided at Section III. Optimal estimation over a sensor tree using a Kalman filter is discussed in Section IV. How to find a tree that uses a minimum transmission energy is described in Section V. This estimate is combined with a linear control law and Section VI shows that the closed-loop system solves an LQG control problem. An example is presented in Section VII to illustrate both the sensor reconfiguration algorithm for finding energy efficient sensor trees and the closed-loop control performance under varying input disturbances. The paper is concluded in Section VIII with a discussion on future work.

\section{Problem Set-Up}

\section{A. Mathematical Models}

Consider the feedback control system in Figure 1. The plant is given by

$$
x_{k}=A x_{k-1}+B u_{k-1}+w_{k-1},
$$

where $(A, B)$ is controllable, $A^{-1}$ exists and $w_{k}$ is white Gaussian noise with zero-mean and covariance matrix $Q_{k} \geq$ $0 .{ }^{1} \mathrm{~A}$ wireless sensor network is used to measure the state. The measurement equation for sensor $S_{i}$ is given by

$$
y_{k}^{i}=H_{i} x_{k}+v_{k}^{i},
$$

where $v_{k}^{i}$ is white Gaussian noise with zero-mean and covariance matrix $\Pi_{i}>0$.

Each sensor can potentially communicate via a single-hop connection with a subset of all the sensors by adjusting its transmission power. Let us introduce a sensor $S_{0}$, which we denote the fusion center, and consider a tree $T$ with root $S_{0}$ (see Figure 2). We suppose that there is a non-zero single-hop communication delay, which is smaller than the sampling

\footnotetext{
${ }^{1}$ We require $A^{-1}$ exists in order to prove certain property of the algorithms presented in Section V. If Eqn (1) is derived by discretizing a continuous process, then $A^{-1}$ is guaranteed to exist.
}

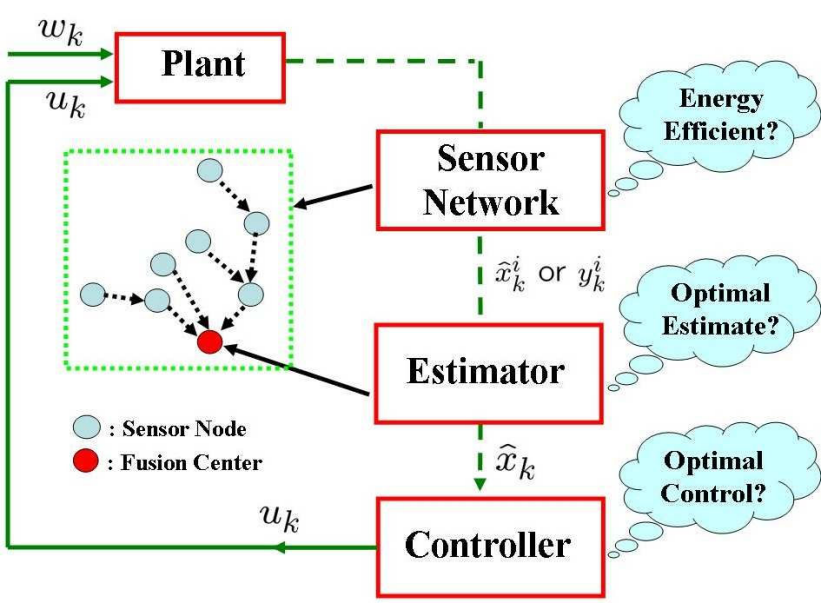

Fig. 1. Structure of Closed-Loop Control System with Measurements Gathered by a Wireless Sensor Tree

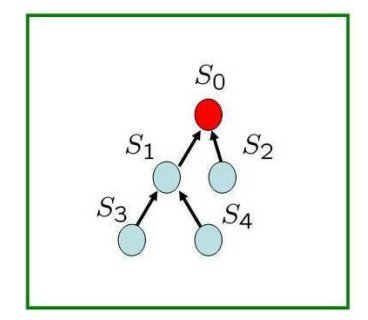

Fig. 2. An Example of a Tree

time of the plant. All sensors are synchronized in time, so the data packet transmitted from $S_{i}$ to $S_{0}$ is delayed one sample when compared with the parent node of $S_{i}$.

\section{B. Problems of Interest}

We are interested in the following problems.

1) Optimal Control Over Sensor Tree: One of the main objectives of the paper is to study how we can close the loop of control over the wireless sensor network. In particular, we pose the standard LQG optimal control problem as follows.

Problem 2.1: Given a tree $T$ representing the sensor communications with $S_{0}$, find the static gain control law $u_{k}=-L \hat{x}_{k}$ that minimizes

$$
J=\lim _{K \rightarrow \infty} \frac{1}{K} \sum_{k=1}^{K} \mathbb{E}\left[x_{k}^{\prime} \Phi x_{k}+u_{k}^{\prime} \Psi u_{k}\right]
$$

where $\Phi \geq 0$ and $\Psi>0$.

2) Optimal Estimation Over Sensor Tree: By the Separation Principle, Solving Problem 2.1 relies on the solution to the following optimal estimation problem.

Problem 2.2: Given a tree $T$ representing sensor communications with $S_{0}$, compute the optimal state estimate $\hat{x}_{k}(T)$.

3) Minimum Energy Routing Tree: Since the network life is largely determined by how fast individual node consumes energy, it is natural to let the network operates at an energy level that is as low as possible, thus we seek the solution to 
the following problem which is rephrased in mathematical terms in Section V.

Problem 2.3: How should the tree $T$ be established such that the total network energy cost is minimum yet the network provides a guaranteed level of estimation accuracy? These three problems are examined in detail in the rest of the paper. We first study the optimal estimation problem as it will form the basis to solve other two problems.

\section{DEFinitions AND KaLman FILTER PRELIMINARIES}

\section{A. Definitions}

Define the following terms for a given a tree $T$ representing sensor communications with $S_{0}$.

- Node $(T)$ : The nodes of $T$, a subset of $\left\{S_{i}\right\}$.

- $\operatorname{Fam}_{T}\left(S_{i}\right)$ : The subtree of $T$ that is rooted at $S_{i}$.

- $\operatorname{Par}_{T}\left(S_{i}\right)$ : The parent node of $S_{i}$ in $T$.

- $\operatorname{Edge}(T)$ : The edges of $T$.

We use the notation $S_{T}=\operatorname{Node}(T)$. Sometimes with write $S_{i} \in T$ to mean $S_{i} \in S_{T}$. The depth of $T$ is denoted $h_{T}$, i.e., the length of the path between $S_{0}$ and the leave of $T$ furthest away. For all notations in the paper, we drop the subscript $T$ when the considered tree follows from the context.We suppose $T$ can be modified by each sensor $S_{i} \neq S_{0}$ via the following two simple operations:

- $S_{i}$ breaks its link to $\operatorname{Par}\left(S_{i}\right)$, i.e., $T:=T \backslash \operatorname{Fam}\left(S_{i}\right)$.

- $S_{i}$ breaks its link to $\operatorname{Par}\left(S_{i}\right)$ and connects to $S_{0}$, i.e., $\operatorname{Par}\left(S_{i}\right):=S_{0}$.

The first operation corresponds to removing the sensors $\operatorname{Fam}\left(S_{i}\right)$ from $T$. The second operation corresponds to reducing the number of hops between the sensors $\operatorname{Fam}\left(S_{i}\right)$ and $S_{0}$. Let $T_{\text {all }}$ denote all trees that these operations can lead to for a given initial tree $T_{0}$. We will provide an algorithm to construct such an initial tree $T_{0}$ rooted at $S_{0}$ in Section VB.1. Note that $T_{\text {all }}$ is typically a strict subset of all trees with root $S_{0}$ and nodes from $S$.

Next, we formalize estimation over a sensor tree under communication energy constraints, in which the operations above can be used to improve the performance.Let us define the following state estimates and other quantities at $S_{0}$ :

$$
\begin{aligned}
\hat{x}_{k}^{-}(T) & \triangleq \mathbb{E}\left[x_{k} \mid \text { all measurements up to } k-1\right], \\
\hat{x}_{k}(T) & \triangleq \mathbb{E}\left[x_{k} \mid \text { all measurements up to } k\right], \\
P_{k}^{-}(T) & \triangleq \mathbb{E}\left[\left(x_{k}-\hat{x}_{k}^{-}(T)\right)\left(x_{k}-\hat{x}_{k}^{-}(T)\right)^{\prime}\right], \\
P_{k}(T) & \triangleq \mathbb{E}\left[\left(x_{k}-\hat{x}_{k}(T)\right)\left(x_{k}-\hat{x}_{k}(T)\right)^{\prime}\right], \\
P_{\infty}^{-}(T) & \triangleq \lim _{k \rightarrow \infty} P_{k}^{-}(T), \text { if the limit exists, } \\
P_{\infty}(T) & \triangleq \lim _{k \rightarrow \infty} P_{k}(T), \text { if the limit exists. }
\end{aligned}
$$

\section{B. Kalman Filter Preliminaries}

Consider the following discrete time system

$$
\begin{aligned}
x_{k} & =A x_{k-1}+w_{k-1} \\
y_{k} & =C_{k} x_{k}+v_{k}
\end{aligned}
$$

where $w_{k-1}$ and $v_{k}$ are white Gaussian noises with zeromean and covariances $Q_{k} \geq 0$ and $R_{k}>0$, respectively.
The estimates $\hat{x}_{k}$ and $P_{k}$ can be computed as

$$
\left(\hat{x}_{k}, P_{k}\right)=\mathbf{K F}\left(\hat{x}_{k-1}, P_{k-1}, y_{k}, C_{k}, Q_{k}, R_{k}\right),
$$

where KF denotes the Kalman filter which is given by

$$
\begin{aligned}
\hat{x}_{k}^{-} & =A \hat{x}_{k-1}, \\
P_{k}^{-} & =A P_{k-1} A^{\prime}+Q_{k}, \\
K_{k} & =P_{k}^{-} C_{k}^{\prime}\left[C_{k} P_{k}^{-} C_{k}^{\prime}+R_{k}\right]^{-1}, \\
\hat{x}_{k} & =A \hat{x}_{k-1}+K_{k}\left(y_{k}-C_{k} A \hat{x}_{k-1}\right), \\
P_{k} & =\left(I-K_{k} C_{k}\right) P_{k}^{-} .
\end{aligned}
$$

It can be shown that $P_{k}^{-}$evolves as

$$
\begin{aligned}
& P_{k}^{-}=A P_{k-1}^{-} A^{\prime}+Q_{k} \\
& -A P_{k-1}^{-} C_{k-1}^{\prime}\left[C_{k-1} P_{k-1}^{-} C_{k-1}^{\prime}+R_{k}\right]^{-1} C_{k-1} P_{k-1}^{-} A^{\prime} .
\end{aligned}
$$

In the case $C_{k}=C, Q_{k}=Q, R_{k}=R$ and the limit exists, $P_{k}^{-}$in steady state satisfies

$$
P_{\infty}^{-}=A P_{\infty}^{-} A^{\prime}+Q-A P_{\infty}^{-} C^{\prime}\left[C P_{\infty}^{-} C^{\prime}+R\right]^{-1} C P_{\infty}^{-} A^{\prime} .
$$

\section{Optimal Estimation Over A Tree}

Let the tree $T$ that represents the sensor communications with $S_{0}$ be given. Recall that $T$ has depth $h$. Thus at time $k, S_{0}$ has the following measurements:

- $Y_{k}^{1} \triangleq\left\{y_{k}^{i}: S_{i}\right.$ is 1 hop away from $\left.S_{0}\right\}$,

- $Y_{k}^{2} \triangleq\left\{y_{k-1}^{i}: S_{i}\right.$ is 2 hop away from $\left.S_{0}\right\}$,

- :

- $Y_{k}^{h} \triangleq\left\{y_{k-h+1}^{i}: S_{i}\right.$ is $h$ hop away from $\left.S_{0}\right\}$.

For example in Figure 2, at time $k, S_{0}$ has measurements $Y_{k}^{1}=\left\{y_{k}^{1}, y_{k}^{2}\right\}, Y_{k}^{2}=\left\{y_{k-1}^{3}, y_{k-1}^{4}\right\}$. We can then define

$$
Y_{k}^{k-i+1} \triangleq Y_{k-i+1}^{1} \bigcup \cdots \bigcup Y_{k}^{i}, i=1, \cdots, h
$$

as all the measurement available for time $k-i+1$ at time $k$. Notice that $Y_{k-1}^{1}$ are the available measurements at time $k-1$. However at time $k$, the available measurements for time $k-1$ changes to $Y_{k}^{k-1}=Y_{k-1}^{1} \bigcup Y_{k}^{2}$. Hence we can obtain a better estimate of $x_{k-1}$ at time $k$ than at time $k-1$. This inspired us to regenerate the optimal estimate of the previous states and use them as input to generate the current estimate. That is the basic idea contained in Theorem 4.1 where we generated the optimal estimate of $x_{k-h+1}, \cdots, x_{k-1}$ at time $k$ and then make use of the updated estimates to generate the current estimate $\hat{x}_{k}$. Similar idea was presented in [16] when estimation over a queuing network was considered. Figure 3 shows the overall estimation scheme at time $k$.

Let $S_{i_{j}}$ be the node that is $j$ hops away from $S_{0}$. Define

$$
\begin{aligned}
& \Gamma_{j} \triangleq\left[H_{1_{j}} ; H_{2_{j}} ; \cdots\right], j=1, \cdots, h \\
& C_{i} \triangleq\left[\Gamma_{1} ; \cdots ; \Gamma_{i}\right], i=1, \cdots, h \\
& \Upsilon_{j} \triangleq \operatorname{diag}\left\{\Pi_{1_{j}}, \Pi_{2_{j}}, \cdots\right\}, j=1, \cdots, h \\
& R_{i} \triangleq \operatorname{diag}\left\{\Upsilon_{1}, \cdots, \Upsilon_{i}\right\}, i=1, \cdots, h
\end{aligned}
$$

For $X \geq 0$, define

$g_{C_{i}}(X) \triangleq A X A^{\prime}+Q-A X C_{i}^{\prime}\left[C_{i} X C_{i}^{\prime}+R_{i}\right]^{-1} C_{i} X A^{\prime}$. 


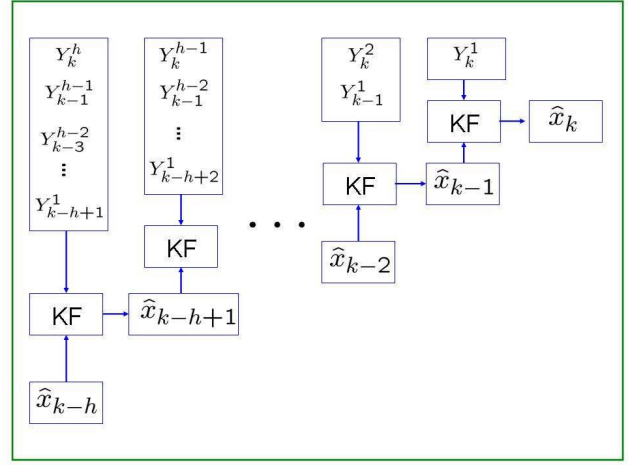

Fig. 3. Kalman Filter Iterations at Time $k$

Theorem 4.1: Consider a sensor tree $T$ with depth $h$.

1) $\hat{x}_{k}$ and $P_{k}$ can be computed from $h$ parallel filter as

$$
\begin{aligned}
& \left(\hat{x}_{k-h+1}, P_{k-h+1}\right) \\
= & \mathbf{K F}\left(\hat{x}_{k-h}, P_{k-h}, Y_{k}^{k-h+1}, C_{h}, Q_{k}, R_{h}\right) \\
\vdots & \\
& \left(\hat{x}_{k-1}, P_{k-1}\right) \\
= & \mathbf{K F}\left(\hat{x}_{k-2}, P_{k-2}, Y_{k}^{k-1}, C_{2}, Q_{k}, R_{2}\right) \\
& \left(\hat{x}_{k}, P_{k}\right) \\
= & \mathbf{K F}\left(\hat{x}_{k-1}, P_{k-1}, Y_{k}^{k}, C_{1}, Q_{k}, R_{1}\right)
\end{aligned}
$$

2) Furthermore $P_{k}^{-}$and $P_{\infty}^{-}$satisfies

$$
\begin{aligned}
& P_{k}^{-}=g_{C_{2}} \cdots \circ g_{C_{h}}\left(P_{k-h}^{-}\right) \\
& P_{\infty}^{-}=g_{C_{2}} \cdots \circ g_{C_{h-1}}\left(\bar{P}_{\infty}^{-}\right)
\end{aligned}
$$

where $\bar{P}_{\infty}$ is the unique solution to $g_{C_{h}}\left(\bar{P}_{\infty}^{-}\right)=\bar{P}_{\infty}^{-}$.

Proof: 1) We know that the estimate $\hat{x}_{k}$ is generated from the estimate of $\hat{x}_{k-1}$ together with all the available measurements at time $k$ through a traditional Kalman filter. Similarly, the estimate $\hat{x}_{k-1}$ is generated from the estimate of $\hat{x}_{k-2}$ together with all the available measurements for time $k-1$ at time $k$, etc. This recursion for $h$ steps corresponds to the parallel filter stated in the theorem.

2) From Eqn (9), $P_{k-i+1}^{-}$satisfies

$$
P_{k-i+1}^{-}=g_{C_{i+1}}\left(P_{k-i}^{-}\right), i=1,2, \cdots, h-1 \text {. }
$$

Hence $P_{k}^{-}$and $P_{\infty}^{-}$satisfy Eqn (10) and (11).

\section{Minimum Energy Communication Over Sensor TREES}

Let us incorporate the cost of communication by defining an optimization problem over the total transmission power used by the sensor tree. Define $E_{i}$ to be the energy cost for $S_{i}$ sending the measurements of $\operatorname{Fam}\left(S_{i}\right)$ to $\operatorname{Par}\left(S_{i}\right)$. The total energy cost for a tree $T$ is denoted as

$$
E(T)=\sum_{S_{i} \in T} E_{i}
$$

The transmission power typically grows rapidly with the distance to the receiver. ${ }^{2}$ Hence, it is desirable to communicate only with close nodes to save energy. On the other hand, low transmission power, leads to many hops between some sensors and the fusion center, which adds delays to the measurements gathered in the fusion center. It is thus natural to seek a tree $T \in T_{\text {all }}$ with desired estimation accuracy $P_{\text {desired }}>0$ and minimum communication energy cost. We rephrase Problem 2.3 as follows

Problem 5.1:

$$
\min _{T \in T_{\text {all }}} E(T)
$$

subject to

$$
\operatorname{Tr}\left(P_{\infty}(T)\right) \leq P_{\text {desired }}
$$

We write $P_{\infty}(T) \leq P_{\text {desired }}$ in the trace sense later. We first analyze the complexity of the problem by seeking the optimal solution, which is shown to be intractable. We then present some heuristic algorithms to tackle the problem, which are efficient but at the price of only producing local optimal solution in general.

\section{A. Optimal Solution Via Exhaustive Search}

Let $N$ be the total number of sensors in $T_{0}$. Then

$$
\left|T_{\text {all }}\right| \approx \sum_{i=1}^{N}\left(\begin{array}{c}
i \\
N
\end{array}\right) 3^{i}=\sum_{i=1}^{N} \frac{N !}{i !(N-i) !} 3^{i},
$$

as a tree in $T_{\text {all }}$ can have $i$ sensors, $1 \leq i \leq N$, and each sensor has the choice of disconnecting to its parent, connecting to its parent or to the fusion center directly (except those sensors that are 1 hop away from $S_{0}$ ). Problem 5.1 is an optimization problem over the discrete set $T_{\text {all }}$. From Eqn (13), it is apparent that the optimal solution via exhaustive search is intractable for large $N$.

\section{B. Tree Reconfiguration Algorithm}

In this section, we present a Tree Reconfiguration Algorithm (Figure 4) which solves Problem 5.1 efficiently. However, the solution from this algorithm may not be the optimal one in general.

The Tree Reconfiguration Algorithm consists of three subroutines. The first subroutine is called by executing the Tree Initialization Algorithm to produce the initial tree $T_{0}$ (the top rectangular block in Figure 4). Depending on whether $T_{0}$ provides enough required accuracy, two other subroutines are called by executing the Switching Tree Topology Algorithm (the middle right rectangular block) and the Minimum Energy Subtree Algorithm(the bottom rectangular block) respectively. These algorithms are presented in details next.

\footnotetext{
${ }^{2}$ An estimate of $E_{i}$ can be be computed based on the considered wireless technology. A common model is that if the distance between $S_{i}$ and $\operatorname{Par}\left(S_{i}\right)$ is $d_{i}$, then $E_{i}=\beta_{i}+\alpha_{i}\left(d_{i}\right)^{n_{i}}$, where $\beta_{i}$ represents the static part of the energy consumption and $\alpha_{i}\left(d_{i}\right)^{n_{i}}$ the dynamic part. The path loss exponent $n_{i}$ is typically between 2 and 6 .
} 


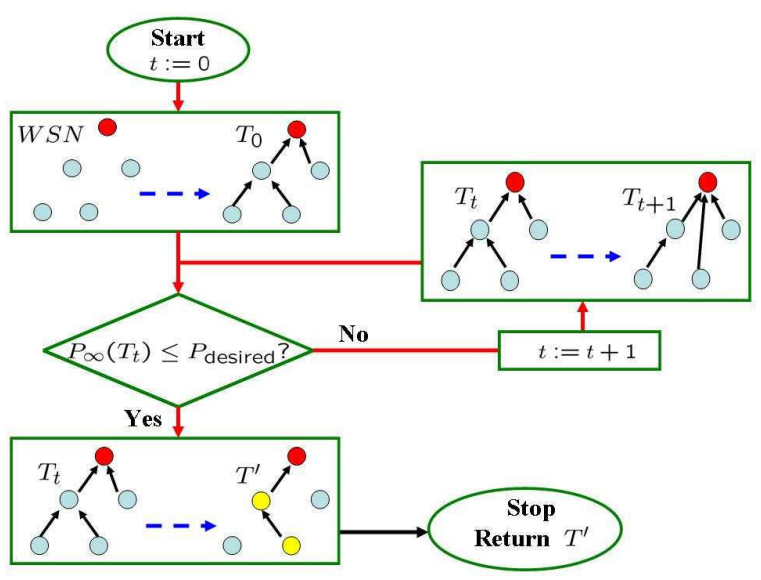

Fig. 4. Tree Reconfiguration Algorithm

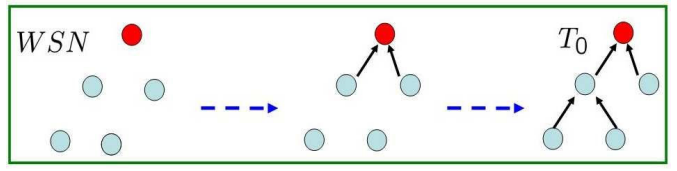

Fig. 5. Tree Initialization Algorithm

1) Tree Initialization Algorithm: Let $T_{0}$ denote the tree which represents the initial connection of the sensors with $S_{0} . T_{0}$ is constructed via the Tree Initialization Algorithm presented graphically in Figure 5. The idea is that $S_{0}$ first establishes direct connections with its neighbor sensors. After that, its neighbor sensors establish further connections with their own neighbor sensors. This process continues until a tree of depth $h_{T}$ is formed. The actual implementation of the algorithms is provided in Section A of the Appendix.

2) Switching Tree Topology Algorithm: For a given tree $T_{t}$, if $P_{\infty}\left(T_{t}\right)>P_{\text {desired }}$, the tree needs to be adjusted in a way that the accuracy is improved. The Switching Tree Topology Algorithm provides such a way.

We assume if $S_{i}$ breaks connection with $\operatorname{Par}\left(S_{i}\right)$ and connects directly to $S_{0}, E_{i}\left(\operatorname{Par}\left(S_{i}\right)\right) \leq E_{i}\left(S_{0}\right)$ and define this operation as $\pi\left(T_{t}, S_{i}\right)$, i.e.,

$\operatorname{Node}\left(\pi\left(T_{t}, S_{i}\right)\right)=\operatorname{Node}\left(T_{t}\right)$ and

$$
\operatorname{Edge}\left(\pi\left(T_{t}, S_{i}\right)\right)=\operatorname{Edge}\left(T_{t}\right) \bigcup\left\{S_{i}, S_{0}\right\} \backslash\left\{S_{i}, \operatorname{Par}_{T_{t}}\left(S_{i}\right)\right\}
$$

Further define $S_{2 \text { hop }} \triangleq\left\{S_{i}: \operatorname{Par}\left(\operatorname{Par}\left(S_{i}\right)\right)=S_{0}\right\}$. The algorithm is then given as follows.

\begin{tabular}{l}
\hline Switching Tree Topology Algorithm \\
- Init: $T_{t}$. \\
- Compute $S_{i}=\arg \min _{S_{i} \in S_{2 \mathrm{hop}}} P_{\infty}\left(\pi\left(T_{t}, S_{i}\right)\right)$. \\
- Return $T_{t+1}:=\pi\left(T_{t}, S_{i}\right)$. \\
\hline
\end{tabular}

3) Minimum Energy Subtree Algorithm: For a given tree $T_{t}$ with $P_{\infty}\left(T_{t}\right) \leq P_{\text {desired }}$, The Minimum Energy Subtree Algorithm finds the subtree $T^{\prime}$ rooted at $S_{0}$ with the property that $P_{\infty}\left(T^{\prime}\right) \leq P_{\text {desired }}$, and $E\left(T^{\prime}\right) \leq E(\tilde{T})$ for any subtree $\tilde{T}$ of $T_{t}$ rooted at $S_{0}$. The idea is that all possible subtrees

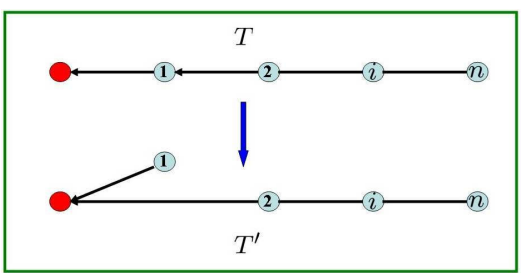

Fig. 6. Switching Tree Topology

$\tilde{T}$ rooted at $S_{0}$ and satisfying

$$
P_{\infty}(\tilde{T}) \leq P_{\text {desired }}
$$

are found in an efficient way utilizing the structure of the initial tree $T_{0}$. Then the subtree $T^{\prime}$ which has the least communication energy is returned. The actual implementation of the algorithm and an example are provided in Section B of the Appendix.

\section{Performance Analysis of the Algorithms}

The performance of the Tree Reconfiguration Algorithm in previous section is summarized in the following theorem.

Theorem 5.2: (1) Given a tree $T$ with $P_{\infty}(T)>P_{\text {desired, }}$, the Switching Tree Topology Algorithm returns $T^{\prime} \in T_{\text {all }}$ such that

$$
P_{\infty}\left(T^{\prime}\right) \leq P_{\infty}(T) \text { and } E\left(T^{\prime}\right) \geq E(T) .
$$

(2) Given a tree $T$ with $P_{\infty}(T) \leq P_{\text {desired }}$, the Minimum Energy Subtree Algorithm returns $T^{\prime} \subset T$ rooted at $S_{0}$ such that

$$
P_{\infty}\left(T^{\prime}\right) \leq P_{\text {desired }} \text { and } E\left(T^{\prime}\right) \leq E(\tilde{T})
$$

for any other $\tilde{T} \subset T$ that is rooted at $S_{0}$.

(3) If $\exists T \in T_{\text {all }}$ such that $P_{\infty}(T) \leq P_{\text {desired }}$, then the output $T^{\prime}$ from the Tree Reconfiguration Algorithm satisfies $P_{\infty}\left(T^{\prime}\right) \leq P_{\text {desired }}$.

Proof: (1) We provide the proof for the line topology (Figure 6). It is straightforward to extend the proof for a general tree. Following the notations in Section IV, for $T$, $P_{\infty}^{-}(T)$ satisfies

$$
P_{\infty}^{-}(T)=g_{C_{2}} \circ g_{C_{3}} \cdots g_{C_{h-1}}\left(\bar{P}_{\infty}^{-}\right),
$$

where $\bar{P}_{\infty}^{-} \geq 0$ is the unique solution to $g_{C_{h}}\left(\bar{P}_{\infty}^{-}\right)=\bar{P}_{\infty}^{-}$ and $C_{i}=\left[H_{1} ; H_{2} ; \cdots H_{i}\right], R_{i}=\operatorname{diag}\left\{\Pi_{1}, \Pi_{2}, \cdots, \Pi_{i}\right\}$ for $i=2, \cdots, h$. For $T^{\prime}, P_{\infty}^{-}\left(T^{\prime}\right)$ satisfies

$$
\begin{aligned}
P_{\infty}^{-}\left(T^{\prime}\right) & =g_{C_{3}} \circ g_{C_{4}} \cdots g_{C_{h-1}}\left(\bar{P}_{\infty}^{-}\right) \\
& =g_{C_{3}} \circ g_{C_{4}} \cdots g_{C_{h-1}} \circ g_{C_{h}}\left(\bar{P}_{\infty}^{-}\right) \\
& \leq g_{C_{2}} \cdots g_{C_{i}} \cdots g_{C_{h-2}} \circ g_{C_{h-1}}\left(\bar{P}_{\infty}^{-}\right) \\
& =P_{\infty}^{-}(T)
\end{aligned}
$$

where the inequality is from Corollary 1.3 in Appendix C. Therefore

$$
\begin{aligned}
P_{\infty}\left(T^{\prime}\right) & =A^{-1}\left(P_{\infty}^{-}\left(T^{\prime}\right)\right) A^{\prime-1}-A^{-1} Q A^{\prime-1} \\
& \leq A^{-1}\left(P_{\infty}^{-}(T)\right) A^{\prime-1}-A^{-1} Q A^{\prime-1} \\
& =P_{\infty}(T) .
\end{aligned}
$$


$E(T) \leq E\left(T^{\prime}\right)$ holds from the assumption.

(2) Suppose $T^{*}=\left(S^{*}, \operatorname{Edge}\left(T^{*}\right)\right)$ is the subtree that has the least energy expenses. Let $\Delta S=S \backslash S^{*}=$ $\left\{S_{i_{1}}, S_{i_{2}}, \cdots, S_{i_{m}}\right\}$ with $i_{1} \leq i_{2} \leq \cdots \leq i_{m}$. Then $\Delta S \subset$ $\mathcal{D}_{r}{ }^{3}$, as $P_{\infty}\left(T^{*}\right) \leq P_{\text {desired }}$. We also have $S\left(i_{1} i_{2}\right) \in \mathcal{D}_{r}$ as

$$
P_{\infty}\left(T \backslash S\left(i_{1} i_{2}\right)\right) \leq P_{\infty}\left(T^{*}\right) \leq P_{\text {desired }}
$$

Similarly, $S\left(i_{1} i_{2} \cdots i_{m}\right) \in \mathcal{D}_{r}$ and so $T^{*}=T \backslash$ $S\left(i_{1} i_{2} \cdots i_{m}\right)$ is returned by the Tree Reconfiguration Algorithm as we assume $T^{*}$ is the subtree that has the least energy expense.

(3) Since $P_{\text {total }} \leq P_{\infty}(T)$ for all $T \in T_{\text {all }}$, if such $T$ exists with $P_{\infty}(T) \leq P_{\text {desired }}$, we have $P_{\text {total }} \leq P_{\infty}(T)$. Suppose at $t=t_{1}, P_{\infty}\left(T_{t_{1}}\right) \leq P_{\text {desired }}$, then it is clear that $P_{\infty}\left(T^{\prime}\right) \leq P_{\text {desired }}$. Otherwise, the Tree Reconfiguration Algorithm continues until direct connections between all sensors with $S_{0}$ are established, in which case $P_{\infty}\left(T_{t}\right)=$ $P_{\text {total }} \leq P_{\text {desired }}$. Hence $P_{\infty}\left(T^{\prime}\right) \leq P_{\text {desired }}$.

\section{Optimal LQG CONTROL OVER ENERGy EFFICIENT WIRELESS SENSOR NETWORK}

The theorem below provides an answer to Problem 2.1.

Theorem 6.1: For a given $T$, let the optimal $\hat{x}_{k}(T)$ and corresponding $P_{\infty}(T)$ be generated as in Theorem 4.1. Then $u_{k}=-L \hat{x}_{k}$ that minimizes $J(T)$ defined in Eqn (3) is given by

$$
u_{k}=-\left[B^{\prime} S_{\infty} B+\Psi\right]^{-1} B^{\prime} S_{\infty} A \hat{x}_{k}
$$

where $S_{\infty} \geq 0$ is the unique solution to

$$
S_{\infty}=A^{\prime} S_{\infty} A+\Phi-A^{\prime} S_{\infty} B\left[B^{\prime} S_{\infty} B+\Psi\right]^{-1} B^{\prime} S_{\infty} A .
$$

Furthermore, $J(T)$ can be computed as

$$
J(T)=\operatorname{Tr}\left(S_{\infty} Q\right)+\operatorname{Tr}\left(F P_{\infty} F^{\prime}\right)
$$

where $F=A^{\prime} S_{\infty} B\left[B^{\prime} S_{\infty} B+\Psi\right]^{-\frac{1}{2}}$.

Proof: Following from [17] and together with Theorem 4.1.

Notice that $S_{\infty}$ is a fixed quantity, hence $\operatorname{Tr}\left(S_{\infty} Q\right)$ is independent of $T$. Also notice that $F P_{\infty} F^{\prime}$ is quadratic in $P_{\infty}$, therefore in order to keep $J(T) \leq J_{\text {desired }}, P_{\infty}$ has to satisfy $P_{\infty} \leq P_{\text {desired }}$, which is the reason we choose the minimum energy subtree subject to the estimation constraint.

\section{EXAMPLE}

We consider an integrator chain as an example in this section. The discrete time system dynamics is given by Eqn (1) with

$$
A=\left[\begin{array}{cccc}
1 & 0.1 & 0.05 & 0.0002 \\
0 & 1 & 0.1 & 0.05 \\
0 & 0 & 1 & 0.1 \\
0 & 0 & 0 & 1
\end{array}\right], B=\left[\begin{array}{l}
0 \\
0 \\
0 \\
1
\end{array}\right]
$$

\footnotetext{
${ }^{3}$ See Section $\mathrm{B}$ of the Appendix for the definition of $\mathcal{D}_{r}$ and $S\left(i_{1} i_{2} \cdots i_{l}\right)$ later in the proof.
}

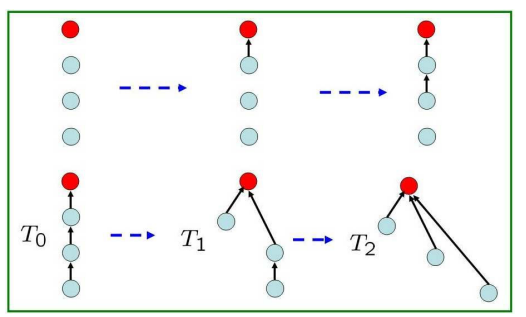

Fig. 7. Different Trees Formed by the Tree Reconfiguration Algorithm

There are three sensors available. The measurement equations are given by

$$
\begin{aligned}
& y_{k}^{1}=\left[\begin{array}{llll}
1 & 0 & 0 & 0
\end{array}\right] x_{k}+v_{k}^{1}, \\
& y_{k}^{2}=\left[\begin{array}{llll}
0 & 1 & 0 & 0
\end{array}\right] x_{k}+v_{k}^{2}, \\
& y_{k}^{3}=\left[\begin{array}{llll}
0 & 0 & 1 & 0
\end{array}\right] x_{k}+v_{k}^{3},
\end{aligned}
$$

where $v_{k}^{i}$ are white Gaussian with zero-mean and covariances $\Pi_{1}=0.25, \Pi_{2}=0.5$ and $\Pi_{3}=0.5$. Assume sensor $i$ is $i$ hops away from $S_{0}$ (Figure 7).

Further assume that if $S_{i}$ is connected to $S_{i-1}, i=1,2,3$, the energy of communication is $e$; if $S_{i}$ is connected to $S_{i-2}, i=2,3$, the energy is $4 e$ and if $S_{3}$ is connected to $S_{0}$, the energy is $8 e$. The control law $u_{k}$ is computed as in Theorem 6.1. Suppose it is required that $\operatorname{Tr}\left(P_{\text {desired }}\right) \leq 10$ for this system. Initially, assume $Q_{k}=Q_{0}$ for all $k \leq$ $k_{1}=200$, where $Q_{0}=0.2 I$. After $T_{0}$ is set up, $S_{0}$ computes $\operatorname{Tr}\left(P_{\infty}\left(T_{0}\right)\right)=4.1297<10$. Thus it starts to run the Minimum Energy Subtree Algorithm to find out $T^{\prime}$. In this case $T^{\prime}=T_{0} \backslash S_{3}$ with $\operatorname{Tr}\left(P_{\infty}\left(T^{\prime}\right)\right)=9.6411$ and $E\left(T^{\prime}\right)=2 e$.

We model the disturbance to the plant as changes to $Q_{k}$. Suppose at time $k_{1}, Q_{k}$ changes to $4 Q_{0}$ and will last for 100 time steps. We assume the changes in $Q_{k}$ is known to $S_{0}{ }^{4}$ In this case, $T_{0} \backslash S_{3}$ no longer provides enough accuracy as $P_{\infty}\left(T^{\prime}\right)$ changes to 34.9300 . Thus $S_{0}$ executes the Tree Reconfiguration Algorithm again to find the desired tree. Now only the star topology $T_{2}$, with $\operatorname{Tr}\left(P_{\infty}\left(T_{2}\right)\right)=9.6369$, provides enough accuracy. The price to pay for reconfiguring to $T_{2}$ is that $E\left(T_{2}\right)=13 e$. Figure 10 shows how the different tree location changes in the $E-P_{\infty}$ diagram for these two scenarios. Later when $Q_{k}$ changes back to $Q_{0}$ at $k_{2}=300$, $T_{2}$ is reconfigured to $T_{0} \backslash S_{3}$ as well.

Figure 8- 9 show the evolution of the first and fourth component of $x_{k}$ and the estimation error $e_{k}$ with and without the tree reconfiguration. As we can see from the lower half of the Figures, the state and the estimation remain almost the same after the tree reconfiguration, while if the tree is kept the same, there is a big fluctuation in the state and the estimation error during the time $Q_{k}$ changes to higher values.

\footnotetext{
${ }^{4}$ In the actual implementation, we can estimate the value of $Q_{k}$ using
} various available schemes (eg, see [18]). 

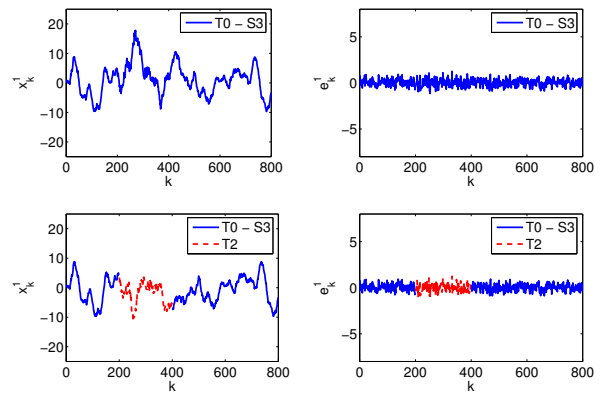

Fig. 8. $x_{k}^{1}$ and $e_{k}^{1}$ with/without Tree Reconfiguration
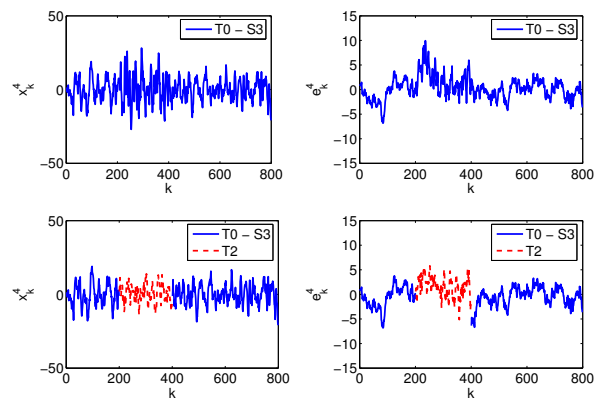

Fig. 9. $x_{k}^{4}$ and $e_{k}^{4}$ with/without Tree Reconfiguration

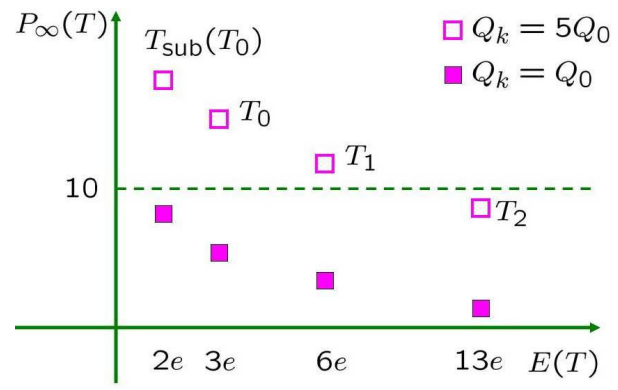

Fig. 10. Tree Location Change in Energy-Accuracy diagram

\section{CONCLUSION AND Future WORK}

In this paper, we have considered an optimal control problem over a wireless sensor network. By dynamically reconfiguring the sensor network, the desired performance can be met minimum energy usage. We present optimal estimation and control schemes over a tree of wireless sensors. We also provide algorithms that seeks the minimum energy subtree.

There are a number of interesting extensions of the current work that we shall pursue in the future. We will include the analysis of the time complexity of the algorithm presented in Section V. We will also explore the case where an estimator of the plant input disturbance $Q_{k}$ is included and see how the uncertainties in estimating $Q_{k}$ relates to the uncertainties in $J(T)$.

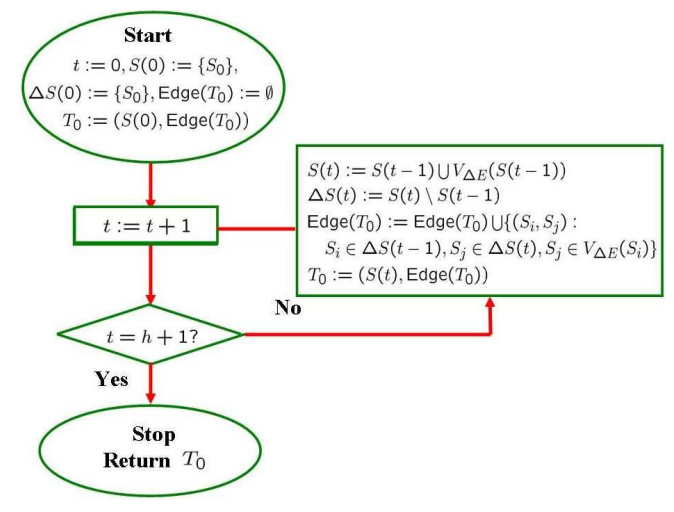

Fig. 11. Tree Initialization Algorithm

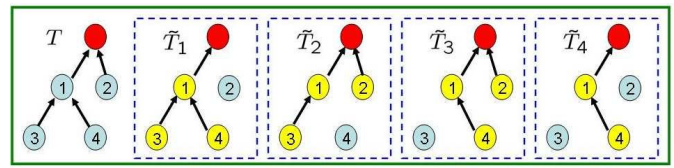

Fig. 12. Tree $T$ and its subtrees $\tilde{T}$ s

\section{APPENDIX}

\section{A. Tree Initialization Algorithm}

We have represented the Tree Initialization Algorithm graphically in Figure 5 in Section V-B.1. In this section, we provide the actual implementation of the algorithm. Define the following quantities.

- $S(t)$ : Sensors added to $T_{0}$ at step $t$.

- $\Delta S(t)$ : Newly added sensors at step $t$.

- $V_{\Delta E}\left(S_{i}\right) \triangleq\left\{S_{j}: S_{j}\right.$ is reachable by $S_{i}$ using $\Delta E$ energy $\}$.

$$
V_{\Delta E}(\Sigma) \triangleq \bigcup_{S_{i} \in \Sigma} V_{\Delta E}\left(S_{i}\right) .
$$

The Tree Initialization Algorithm is presented in its flow diagram form in Figure 11.

\section{B. Minimum Energy Subtree Algorithm}

We present the Minimum Energy Subtree Algorithm with mathematical detail here. To make the presentation clear and easy to follow, we divide the algorithm into several key steps and provide an example to illustrate each step. Before we introduce the algorithm, let us define

- $S\left(i_{1} i_{2} \cdots i_{l}\right) \triangleq\left\{S_{i_{1}}, S_{i_{2}}, \cdots S_{i_{l}}\right\}$.

- $\Omega\left(i_{1} i_{2} \cdots i_{l}\right) \triangleq T \backslash S\left(i_{1} i_{2} \cdots i_{l}\right)$.

where it is assumed $i_{1} \leq i_{2} \leq \cdots \leq i_{l}$. The example we will use is given as follows.

Example 1.1: Consider the tree $T$ with 4 sensor nodes in Figure 12. Assume the following:

1) $T$ provides enough estimation accuracy, i.e., $P_{\infty}(T) \leq$ $P_{\text {desired. }}$.

2) No single sensor provides enough estimation accuracy, i.e., $P_{\infty}(S(i))>P_{\text {desired }}, i=1,2,3,4$. 
3) Among the two sensor pairs, only sensor 1 and 4 can provide enough estimation accuracy, i.e., $P_{\infty}(S(i j)) \leq$ $P_{\text {desired }}$ iff $\{i, j\}=\{1,4\}$.

4) Any three sensors except $\left(S_{2}, S_{3}, S_{4}\right)$ can provide enough estimation accuracy, i.e., $P_{\infty}(\Omega(i)) \leq P_{\text {desired }}, i=$ $2,3,4$.

5) The energy cost of single hop communication is $e$.

By the above assumptions, it is easy to see that the minimum energy subtree $T^{\prime}$ is given by $\tilde{T}_{4}$ with $E\left(T^{\prime}\right)=2 e$.

Let us examine the case when we take $T$ as an input to the Minimum Energy Subtree Algorithm which consists of the following key steps.

\begin{tabular}{l} 
Step 1 \\
$\bullet$ Init: $T$ \\
$\bullet t:=0, \mathcal{D}_{t}:=\left\{S_{i_{p}} \in T: P_{\infty}\left(\Omega\left(i_{p}\right)\right) \leq P_{\text {desired }}\right\}$ \\
\hline
\end{tabular}

In this step, $\mathcal{D}_{0}$ holds all single sensor node without which, the rest sensors still satisfy the accuracy requirement. Therefore in Example 1.1 $\mathcal{D}_{0}=\left\{S_{2}, S_{3}, S_{4}\right\}$.

$$
\begin{aligned}
& \text { Step } 2 \\
& \text { - } t:=t+1, \mathcal{D}_{t}:=\mathcal{D}_{t-1} \\
& \text { - } \forall S_{i_{p}} \in \mathcal{D}_{t-1} \text { with } P_{\infty}\left(\Omega\left(i_{p}\right)\right)<P_{\text {desired }} \\
& \quad-\forall q>p \text { and } S_{i_{q}} \notin \operatorname{Fam}\left(S_{i_{p}}\right) \\
& \quad \text { if } P_{\infty}\left(\Omega\left(i_{p} i_{q}\right)\right) \leq P_{\text {desired }}, \mathcal{D}_{t}:=\mathcal{D}_{t} \cup S\left(i_{p} i_{q}\right)
\end{aligned}
$$

In this step, $\mathcal{D}_{1}$ holds all single sensor or two sensor pair without which, the rest sensors still satisfy the accuracy requirement. The third line of step 2 eliminates the redundancy in listing the subtrees as $S\left(i_{p} i_{q}\right)=S\left(i_{q} i_{p}\right)$, and if $S_{i_{p}}$ is removed from a tree, so is $\operatorname{Fam}\left(S_{i_{p}}\right)$. Therefore in Example $1.1 \mathcal{D}_{1}=\left\{S_{2}, S_{3}, S_{4}, S(23)\right\}$.

$$
\begin{aligned}
& \text { Step } 3 \\
& \text { - } t:=t+1, \mathcal{D}_{t}:=\mathcal{D}_{t-1} \\
& \text { - } \forall S\left(i_{p} i_{q}\right) \in \mathcal{D}_{t-1} \text { with } P_{\infty}\left(\Omega\left(i_{p} i_{q}\right)\right)<P_{\text {desired }} \\
& -\forall l>q \text { and } S_{i_{l}} \notin\left(\operatorname{Fam}\left(S_{i_{p}}\right) \cup \operatorname{Fam}\left(S_{i_{q}}\right)\right) \\
& \text { if } P_{\infty}\left(\Omega\left(i_{p} i_{q} i_{l}\right)\right) \leq P_{\text {desired }} \\
& \mathcal{D}_{t}:=\mathcal{D}_{t} \bigcup S\left(i_{p} i_{q} i_{l}\right)
\end{aligned}
$$

Similar to step $3, \mathcal{D}_{2}$ holds all single sensor, two sensor pair or three sensor group without which, the rest sensors still satisfy the accuracy requirement. The algorithm continues in this way until $\mathcal{D}_{r}=\mathcal{D}_{r-1}$ at step $r \leq h$.

\begin{tabular}{|l|}
\hline Step r \\
- Return $T^{\prime}=\arg \min _{\Omega(\cdot) \in \mathcal{D}} E(\Omega(\cdot))$
\end{tabular}

In Example 1.1, $\mathcal{D}_{2}=\left\{S_{2}, S_{3}, S_{4}, S(23)\right\}=\mathcal{D}_{1}$. Hence the algorithm stops and returns $T^{\prime}=\Omega(23)=S(14)=\tilde{T}_{4}$ with $P_{\infty}\left(T^{\prime}\right) \leq P_{\text {desired }}$ and $E\left(T^{\prime}\right)=2 e$. It is easy to verify that $\left|T_{\text {all }}\right|=25$ in this case and the algorithm only calculates 7 of them. In general, the time complexity of executing the Minimum Energy Subtree Algorithm is significantly less than solving Problem 5.1 via exhaustive search.

\section{Some Background}

Let $C_{i}, R_{i}, g_{C_{i}}(X)$ be defined as

$$
\begin{aligned}
C_{i} & \triangleq\left[\Gamma_{1} ; \cdots ; \Gamma_{i}\right], i=1, \cdots, h \\
R_{i} & \triangleq \operatorname{diag}\left\{\Upsilon_{1}, \cdots, \Upsilon_{i}\right\}, i=1, \cdots, h \\
g_{C_{i}}(X) & \triangleq A X A^{\prime}+Q-A X C_{i}^{\prime}\left[C_{i} X C_{i}^{\prime}+R_{i}\right]^{-1} C_{i} X A^{\prime} .
\end{aligned}
$$

Lemma 1.2: Let $1 \leq i \leq j \leq h$ be given. Then

$$
C_{i}^{\prime}\left[C_{i} P C_{i}^{\prime}+R_{i}\right]^{-1} C_{i} \leq C_{j}^{\prime}\left[C_{j} P C_{j}^{\prime}+R_{j}\right]^{-1} C_{j}
$$

Proof: The proof is omitted due to space limitation. Corollary 1.3: For all $i=1, \cdots, n-1$, and all $X \geq 0$,

$$
g_{C_{i+1}}(X) \leq g_{C_{i}}(X) \text {. }
$$

\section{REFERENCES}

[1] H. Kopetz, "Should responsive systems be event triggered or time triggered?" IEICE Trans. on Information and Systems, vol. E76-D(10), pp. $1525-1532,1993$.

[2] K. J. Åström and B. Bernhardsson, "Comparison of periodic and event based sampling for first-order stochastic systems," vol. J. In Preprints 14th World Congress of IFAC, Beijing, P.R. China, pp. 301-306.

[3] L. Bao, M. Skoglund, and K. H. Johansson, "Encoder-decoder design for event-triggered feedback control over bandlimited channels." American Control Conference, Minneapolis, Minnesota, USA, 2006.

[4] D. Siljak, Large-Scale Dynamic Systems: Stability and Structure. North-Holland, New York, 1978.

[5] P. Alriksson and A. Rantzer, "Distributed kalman filtering using weighted averaging." Proc. 17th International Symposium on Mathematical Theory of Networks and Systems, Kyoto, Japan, 2006.

[6] R. Olfati-Saber, "Distributed kalman filter with embedded consensus filters." Proceedings of the 44th IEEE Conference on Decision and Control and European Control Conference, 2005.

[7] D. Spanos, R. Olfati-Saber, and R. M. Murray, "Distributed kalman filtering in sensor networks with quantifiable performance." Proceedings of the 4th International Conference on Information Processing in Sensor Networks, 2005.

[8] B.Sinopoli, L.Schenato, M.Franceschetti, K.Poolla, M.Jordan, and S.Sastry, "Kalman filtering with intermittent observations," IEEE Transactions on Automatic Control, vol. 49, no. 9, pp. 1453-1464, 2004.

[9] J. Hespanha, P. Naghshtabrizi, and Y. Xu, "Networked control systems: Analysis and design," To appear in the Proc. of IEEE, Special Issue on Networked Control Systems, 2007.

[10] D. P. Bertsekas and R. Gallager, Data Networks, 2nd ed. Prentice Hall, 1991.

[11] F. Z. Juan Liu and D. Petrovic, "Information-directed routing in ad hoc sensor networks," IEEE JOURNAL ON SELECTED AREAS IN COMMUNICATIONS, vol. 23, no. 4, April 2005.

[12] A. Bonivento, C. Fischione, and A. Sangiovanni-Vincentelli, "Randomized protocol stack for ubiquitous networks in indoor environment," IEEE CCNC, vol. 1, April 2006.

[13] W. Lai and I. C. Paschalidis, "Routing through noise and sleeping nodes in sensor networks: latency vs. energy trade-offs." Proceedings of the 45th IEEE Conference on Decision and Control, San Diego, CA, USA, Dec 2006, pp. $551-555$.

[14] E. Witrant, P. G. Park, M. Johansson, C. Fischione, and K. H. Johansson, "Predictive control over wireless multi-hop networks." IEEE Conference on Control Applications, Singapore, 2007. Submitted.

[15] A. Panousopoulou, G. Nikolakopoulos, A. Tzes, and J. Lygeros, "Experimental evaluation of a mobile ad-hoc networked (manet)controlled system." Proc. 17th International Symposium on Mathematical Theory of Networks and Systems, Kyoto, Japan, 2006.

[16] M. Epstein, L. Shi, A. Tiwari, and R. M. Murray, "Estimation of linear stochastic systems over a queueing network." International Conference on Sensor Networks, 2005.

[17] K. J. Åström and B. Wittenmark, Computer Controlled Systems. Prentice Hall, 1984.

[18] R. K. Mehra, "On the identification of variances and adaptive kalman filtering," IEEE Transactions on Automatic Control, vol. 15, no. 2, April 1970. 